\title{
Collision-model-based approach to non-Markovian quantum dynamics
}

\author{
F. Ciccarello, ${ }^{1,2}$ G. M. Palma, ${ }^{2}$ and V. Giovannetti ${ }^{1}$ \\ ${ }^{1}$ NEST, Scuola Normale Superiore and Istituto Nanoscienze-CNR, Piazza dei Cavalieri 7, I-56126 Pisa, Italy \\ ${ }^{2}$ NEST, Istituto Nanoscienze-CNR and Dipartimento di Fisica e Chimica, Università degli Studi di Palermo, \\ via Archirafi 36, I-90123 Palermo, Italy
}

(Received 1 August 2012; revised manuscript received 15 December 2012; published 25 April 2013)

\begin{abstract}
We present a theoretical framework to tackle quantum non-Markovian dynamics based on a microscopic collision model (CM), where the bath consists of a large collection of initially uncorrelated ancillas. Unlike standard memoryless CMs, we endow the bath with memory by introducing interancillary collisions between next system-ancilla interactions. Our model interpolates between a fully Markovian dynamics and the continuous interaction of the system with a single ancilla, i.e., a strongly non-Markovian process. We show that in the continuous limit one can derive a general master equation, which, while keeping such features, is guaranteed to describe an unconditionally completely positive and trace-preserving dynamics. We apply our theory to an atom in a dissipative cavity for a Lorentzian spectral density of bath modes, a dynamics which can be exactly solved. The predicted evolution shows a significant improvement in approaching the exact solution with respect to two well-known memory-kernel master equations.
\end{abstract}

DOI: 10.1103/PhysRevA.87.040103

Introduction. In open system dynamics the focus is on a system $S$ in contact with an environment. Typically, the goal is to seek a master equation (ME) where the degrees of freedom (DOF) of $S$ are the only explicit variables. Hence, the environmental interactions should be accounted for through an effective but reliable description. When it comes to quantum objects, this problem turns out to be especially thorny [1-4]. Within this context, "reliable" means that the ME to be worked out should give rise to a completely positive and tracepreserving (CPT) dynamics. It is well assessed that Markovian, i.e., memoryless, environments are described by MEs in the so-called Lindblad form [1] entailing unconditionally CPT dynamics. Markovianity is in most cases only an approximation, though: In general, the environment is not forgetful and there is indeed a broad variety of actual phenomena featuring strong non-Markovian (NM) effects [5]. Yet, a general systematic framework for describing these has yet to be developed. Rather, many different approaches have been proposed. Typically, they rely on phenomenological assumptions and/or approximations (testifying the formidable hurdles to cope with). Thereby, non-CPT-i.e, unphysical-dynamics can occur in certain regimes [6]. Among these descriptive tools are the so-called memory-kernel MEs, e.g., those in Refs. [7,8]. These are integrodifferential MEs featuring a history integral, where past states of $S$ are weighted through a certain memory-kernel function (MKF). There exist regimes in which such MEs can fail to be CPT [9-15]. Moreover, it was recently tested [16] whether MEs in Refs. [7,8] are non-Markovian through a non-Markovianity indicator proposed by Breuer et al. [17]. It turned out that this is null [16], suggesting that such MEs should rather be regarded as time-dependent Markovian. This means that when they entail a CPT dynamics this is anyway very close to the purely Markovian regime (weak non-Markovianity).

Here, we tackle the problem to derive a NM ME through a suitable collision model (CM) [18-25]. This allows us to identify a class of MEs featuring two attractive properties that rarely hold simultaneously. First, they unconditionally fulfill the CPT condition. Second, they nicely allow to interpolate
PACS number(s): 03.65.Yz, 03.67.-a, 42.50.Lc

between the purely Markovian regime and the strongly nonMarkovian situation where $S$ is continuously interacting with a low-dimensional, hence nonforgetful, bath. Also, the model applies regardless of the dimensionality of $S$ and the form of the system-ancilla coupling. We recall that in standard CMs the bath is modeled as a large collection of noninteracting identical ancillas. By hypothesis, $S$ "collides" with each of these one at a time and, importantly, is not allowed to interact more than once with a given ancilla. Demonstrably, such a process gives rise to an irreversible dynamics for $S$ corresponding to a Lindblad-type [1], i.e., Markovian, ME [21]. This can be expected since, as stressed, at each step $S$ comes into contact with a fresh ancilla which is still in its initial state. Hence, there is no way for the bath to keep track of the system's past history. Although they are somewhat fictitious, the latest research is unveiling the potential of CMs as effective theoretical tools for tackling open system dynamics $[24,25]$. First, they are conceptually intuitive, hence potentially easier to cope with: A complex coupling to a large environment is decomposed as a succession of elementary interactions with its subparts. A key feature is that CMs lead to Lindblad-type MEs without demanding any approximation: Only the passage to the continuous limit is needed [21]. This is in contrast to standard microscopic system-reservoir models [1], where Markovianity must be somehow enforced through drastic assumptions such as the requirement of small coupling and short enough bath correlation time (Born-Markov approximation). Should such a feature be maintained in a NM generalization of a CM, this would be quite appealing: As stressed above, approximations and phenomenological assumptions can lead to unphysical predictions. Progress along this line was made recently [22,24,25]. In particular, it was shown [25] that, by taking the ancillas initially in a nontrivial quantum-correlated state, any indivisible channel [1] (thus highly NM) can be simulated when $S$ is a qubit [26]. Here, we tackle the problem from quite a different perspective: Following physical intuition, we describe the memory effects as arising directly from the bath internal dynamics itself. Specifically, in the spirit of standard CMs, a natural memory 
mechanism to devise is adding interancillary (AA) collisions between next system-ancilla (SA) ones. This way, quantum information received from $S$ can be conveyed across the bath and returned to $S$ in the next SA collisions (information backflow): we introduce a $\mathrm{CM}$ with memory precisely built upon this idea.

Model. In a standard (Markovian) CMs [see Fig. 1(a)] collisions $S-1, S-2, S-3$, etc., occur in succession. As each ancilla is still in the initial state before colliding with $S$, memory effects cannot take place. Differently [see Fig. 1(b)], between collisions $S$ - $i$ and $S$ - $(i+1)$, we assume an extra AA collision involving ancillas $i$ and $(i+1)$. This way, before colliding with $S$, the $i$ th ancilla is in a state depending on $S$ 's past history. Any collision is described by a CPT quantum map. Specifically, an SA collision is defined by the $\operatorname{map} \sigma \rightarrow \mathcal{U}_{S i}[\sigma]=\hat{U}_{S i} \sigma \hat{U}_{S i}^{\dagger}$ with $\hat{U}_{S i}=e^{-i \hat{H}_{S i} \tau}$ a unitary operator depending on the collision time $\tau$ and the interaction Hamiltonian $\hat{H}_{S i}$ (we set $\hbar=1$ throughout). Instead, an AA collision between ancillas $i$ and $(i+1)$ is defined as the nonunitary map $\mathcal{S}_{i+1, i}$ which, with probability $p$, exchanges their states. This reads

$$
\sigma \rightarrow \mathcal{S}_{i+1, i}[\sigma]=(1-p) \sigma+p \hat{S}_{i+1, i} \sigma \hat{S}_{i+1, i}
$$

where $\hat{S}_{i+1, i}$ is the swap operator [26] on ancillas $i$ and $i+1$. Different AA collision mechanisms can be selected [27]: The one in Eq. (1) yet has the advantage to allow for simple treatment while fully capturing the idea of environment-mediated information backflow. Here, $p$ plays the role of a knob for tuning the bath memory. The $n$ th-step overall state thus reads $\sigma_{n}=\left(\mathcal{U}_{S n} \circ \mathcal{S}_{n, n-1} \circ \cdots \circ \mathcal{U}_{S 2} \circ \mathcal{S}_{2,1} \circ \mathcal{U}_{S 1}\right)\left[\sigma_{0}\right]$, where "॰" represents the superoperator composition and will be henceforth omitted, and $\sigma_{0}=\rho_{0}|\mathbf{0}\rangle_{B}\langle\mathbf{0}|$ is the system-bath initial state [28] with $\rho_{0}$ being the input density matrix of $S$ and $|\mathbf{0}\rangle_{B}=|0\rangle_{1}|0\rangle_{2} \ldots$ the initial ancillary state [29]. Exploiting the properties of the swap operator and the translational symmetry of $B$ 's initial state, $\sigma_{n}$ can be straightforwardly cast as a sum of terms involving $\left\{\sigma_{m<n}\right\}$. For $n \geqslant 2$, this reads [30]

$$
\sigma_{n}=(1-p) \sum_{j=1}^{n-1} p^{j-1} \mathcal{U}_{S n}^{j}\left[\sigma_{n-j}\right]+p^{n-1} \mathcal{U}_{S n}^{n}\left[\sigma_{0}\right]
$$

(a)

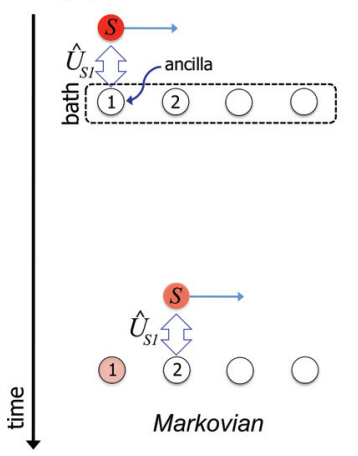

(b)

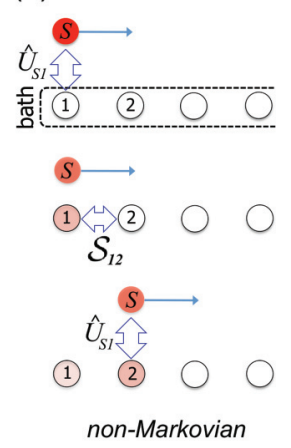

FIG. 1. (Color online) First steps of the process in the memoryless case (a) and in the NM one (b). Next steps are obtained by a mere iteration. where $\mathcal{U}_{S n}^{j}[\sigma]=e^{-i \hat{H}_{S i} j \tau} \sigma e^{i \hat{H}_{S i} j \tau}$. Note that this map corresponds to a coherent interaction between $S$ and the $n$th ancilla only, which continued for a time $j \tau$. This and the fact that in Eq. (2) each $\mathcal{U}_{S n}^{j}$ is applied to $\sigma_{n-j}$ (with $n$ still in $|0\rangle_{n}$ ) entail the attractive property that an expansion for $\rho_{n}=\operatorname{Tr}_{B} \sigma_{n}$ similar to Eq. (2) holds. Tracing this over $B$ indeed yields

$$
\rho_{n}=(1-p) \sum_{j=1}^{n-1} p^{j-1} \mathcal{E}_{j}\left[\rho_{n-j}\right]+p^{n-1} \mathcal{E}_{n}\left[\rho_{0}\right],
$$

where a transformation $\mathcal{E}_{j}$ is a CPT map on $S$ only defined in terms of the unitary map $\mathcal{U}_{S n}^{j}$ and the initial bath state as

$$
\mathcal{E}_{j}[\rho]=\operatorname{Tr}_{B}\left\{\mathcal{U}_{S n}^{j}\left[\rho \otimes|\mathbf{0}\rangle_{\mathbf{B}}\langle\mathbf{0}|\right]\right\}
$$

Interestingly, the structure of Eq. (3) shares features with the discrete model used by Shabani and Lidar (SL) [8] to derive their ME (there, in particular, $\mathcal{E}_{j}$ is the dynamical map in the absence of measurements performed on the bath). Two major differences occur, though. First, Eq. (3) cannot be written as a single sum due to the missing $(1-p)$ factor in the last term, which in fact means that here we deal with a timeinhomogeneous MKF. Second, map $\mathcal{E}_{j}$ is in general strongly NM: It describes the reduced dynamics of $S$ for a continuous coherent interaction between $S$ and a single ancilla (e.g., once can think of two coupled spins periodically exchanging an excitation). Indeed, as anticipated, our model interpolates between two extreme regimes depending on $p$. When $p=0$, AA collisions are absent [cf. Eq. (1)]: Eq. (3) reduces to $\rho_{n}=$ $\mathcal{E}_{1}\left[\rho_{n-1}\right]$ and we retrieve a standard Markovian CM [18-21]. Quite differently, for $p=1$, Eq. (3) yields $\rho_{n}=\mathcal{E}_{n}\left[\rho_{0}\right]$, i.e., $S$ behaves as if it interacts with a single ancilla all the time. This is because for $p=1 \mathrm{Eq}$. (1) reduces to a perfect swap: Once $S$ has collided with $i$, the final state of $i$ is fully transferred to $i+1$ (with $i$ returning to $|0\rangle_{i}$ ).

Master equation. As our next goal, we work out the ME corresponding to Eq. (3) in the continuous limit and prove that (i) it is still capable to interpolate between the two aforementioned opposite limits and (ii) it unconditionally satisfies the CPT condition. For this aim, we first subtract from Eq. (3) the analogous identity for $n-1$. This yields the equation for the variation of $\rho_{n}$ between two next steps $\Delta \rho_{n}=\rho_{n}-\rho_{n-1}$,

$$
\begin{aligned}
\Delta \rho_{n}= & q \sum_{j=1}^{n-2} p^{j-1} \mathcal{E}_{j}\left[\Delta \rho_{n-j}\right]+q p^{n-1} \mathcal{E}_{n-1}\left[\rho_{1}\right] \\
& +\Delta\left(p^{n-1} \mathcal{E}_{n}\right)\left[\rho_{0}\right]
\end{aligned}
$$

with $q=1-p$. This can now be transformed into a differential equation for the continuous-time evolved $S$ state $\rho(t)$ by taking the limit of infinite collisions $(n, j \rightarrow \infty)$ while sending the collision time to zero (i.e., $\tau \rightarrow 0$ ) in such a way that the times $t=n \tau$ and $t^{\prime}=j \tau$ remain finite. Also, when $j$ becomes very large, the probability $p^{j}$ of multiple AA collisions clearly must not vanish. We thus set $p=\exp [-\Gamma \tau]$, where $\Gamma=-(\ln p) / \tau$ is interpreted as the memory rate. We require that, when $\tau \rightarrow 0, p$ approaches 1 in a way that $\Gamma$ stays finite. This allows to express each power of $p$ as a decaying exponential $p^{j}=\left(p^{\frac{1}{\tau}}\right)^{j \tau}=e^{-\Gamma t^{\prime}}$. Note that in the continuous limit $\tau$ should be far shorter than any 
characteristic time, in particular, $\Gamma^{-1}$. Hence, we have $\Gamma \tau \ll 1$ and $1-p=1-e^{-\Gamma \tau} \simeq \Gamma \tau$. Using this, the sum over $j$ in Eq. (5) becomes a time integral as $\tau \rightarrow 0$. By identifying $\Delta \rho_{n} / \tau \rightarrow \dot{\rho}(t)=d \rho(t) / d t$, after a few steps [30] we end up with

$$
\dot{\rho}(t)=\Gamma \int_{0}^{t} d t^{\prime} e^{-\Gamma t^{\prime}} \mathcal{E}\left(t^{\prime}\right)\left[\dot{\rho}\left(t-t^{\prime}\right)\right]+e^{-\Gamma t} \dot{\mathcal{E}}(t)\left[\rho_{0}\right],
$$

where the CPT map $\mathcal{E}(t)$ is the continuous analog of Eq. (4) (the dot stands for the total derivative). This is an integrodifferential ME in $\rho(t)$ featuring a history integral term with an associated MKF $\Gamma e^{-\Gamma t^{\prime}}$ and, notably, a term $\sim \rho_{0}$. The latter is a strong signature of NM behavior. Indeed, in the infinite-memory-time limit $\Gamma \rightarrow 0$, it is the only term surviving in Eq. (5) yielding $\dot{\rho}(t) \rightarrow \dot{\mathcal{E}}(t)\left[\rho_{0}\right]$, i.e., $\rho(t) \rightarrow \mathcal{E}(t)\left[\rho_{0}\right]$ in full analogy with the discrete model (we address the opposite limit $\Gamma \rightarrow \infty$ later on). Next, we derive the solution of Eq. (5), $\rho(t)=\Lambda(t)\left[\rho_{0}\right]$, and prove that the dynamical map [1] $\Lambda(t)$ is always CPT $[\Lambda(0)=\mathcal{I}$ with $\mathcal{I}$ the identity superoperator]. Clearly, $\Lambda(t)$ obeys Eq. (5) under the formal replacement $\rho \rightarrow \Lambda$. Taking its Laplace transform (LT), such an equation is easily solved as [30]

$$
\tilde{\Lambda}(s)=\frac{\tilde{\mathcal{E}}(s+\Gamma)}{\mathcal{I}-\Gamma \tilde{\mathcal{E}}(s+\Gamma)},
$$

where $\tilde{\Lambda}(s)$ and $\tilde{\mathcal{E}}(s)$ are the LTs of $\Lambda(t)$ and $\mathcal{E}(t)$, respectively. Expanding Eq. (6) in powers of $\Gamma$ gives $\tilde{\Lambda}(s)=\sum_{k=1}^{\infty}[\tilde{\mathcal{E}}(s+$ $\Gamma)]^{k} \Gamma^{k-1}$, whose inverse LT is

$$
\Lambda(t)=\mathcal{L}^{-1}[\tilde{\Lambda}(s)](t)=\sum_{k=1}^{\infty} \Gamma^{k-1} \mathcal{L}^{-1}\left[\tilde{\mathcal{E}}^{k}(s+\Gamma)\right](t) .
$$

The basic properties of LT allow to write [30]

$$
\begin{aligned}
\mathcal{L}^{-1}\left[\tilde{\mathcal{E}}^{k}(s+\Gamma)\right]= & e^{-\Gamma t} \int_{0}^{t} d t_{1} \int_{0}^{t_{1}} d t_{2} \cdots \int_{0}^{t_{k-2}} d t_{k-1} \\
& \times \mathcal{E}\left(t_{k-1}\right) \mathcal{E}\left(t_{k-2}-t_{k-1}\right) \cdots \mathcal{E}\left(t-t_{1}\right) .
\end{aligned}
$$

We have thus expressed $\Lambda(t)$ as a weighted series of multiple autoconvolutions of the CPT map $\mathcal{E}(t)$. The integrand in Eq. (8) is evidently a composition of CPT $\mathcal{E}$ maps, thus it is CPT itself. Hence, we see that $\Lambda(t)$ in Eq. (7) is in fact a combination of CPT maps with positive weights [factors $\Gamma^{k-1}$ and $e^{-\Gamma t}$ in Eqs. (7) and (8) are all positive]. This proves that map $\Lambda(t)$ is completely positive. Also, the state obtained by applying the integrand in Eq. (8) (a CPT map as discussed) to $\rho_{0}$ has evidently a unitary trace. As is easily checked [30], this entails $\operatorname{Tr}\left\{\Lambda(t)\left[\rho_{0}\right]\right\}=1$. We conclude that, since $\mathcal{E}(t)$ is CPT, $\Lambda(t)$ is CPT. We finally prove that, in line with Eq. (3) for $p=0$, the Markovian behavior arises from Eq. (5) for $\Gamma \rightarrow \infty$. Indeed, Eq. (5) is such that for $\Gamma$ high enough we can approximate $\mathcal{E}(t) \simeq \mathcal{I}+\mathcal{F} t$, where $\mathcal{F}=\mathcal{E}(0)$. Under LT, this becomes $\tilde{\mathcal{E}}(s)=1 /(s+\Gamma)+$ $\mathcal{F} /(s+\Gamma)^{2}$, which, once plugged into Eq. (6) and for $\Gamma \rightarrow$ $\infty, \quad$ yields $\quad \tilde{\Lambda}(s)=(s+\Gamma+\mathcal{F}) /\left.\left[s^{2}+\Gamma(s-\mathcal{F})\right]\right|_{\Gamma \rightarrow \infty}=$ $1 /(s-\mathcal{F})$. Transforming back, we end up with $\Lambda(t)=e^{\mathcal{F} t}$ entailing that the semigroup property is fulfilled and thus, necessarily, $\mathcal{F}$ is a Lindbladian superoperator [1] with Eq. (5) reducing to the Lindblad form $\dot{\rho}(t)=\mathcal{F}[\rho]$. Interestingly, unlike the SL ME [8], Eq. (5) can yield a Markovian dynamics even for finite $\Gamma$ with a proper choice of map $\mathcal{E}(t)$. Indeed, if $\mathcal{E}(t)=e^{\mathcal{F} t}$, then $\Lambda(t)=e^{\mathcal{F} t}$ is the exact solution of Eq. (5) for any $\Gamma$.

An application. To test the predictive power of our approach, we consider the dynamics of a two-level atom [whose ground (excited) state is denoted by $|0\rangle_{S}\left(|1\rangle_{S}\right)$ ] coupled to a continuum of electromagnetic modes in the rotating-wave approximation [1]. The case in which the field spectral density $J(\omega)$ is a Lorentzian centered on the atomic frequency can be solved exactly [31], which makes it a useful benchmark to assess the effectiveness of a ME [11]. This solution can be expressed in terms of an amplitude damping channel (ADC) [26] as $\rho(t)=\mathcal{A}_{G(t)}\left[\rho_{0}\right]$, where $\mathcal{A}_{\eta}\left[\rho_{0}\right]=$ $\left(1-p|\eta|^{2}\right)|0\rangle_{S}\left\langle\left. 0|+p| \eta\right|^{2} \mid 1\right\rangle_{S}\langle 1|+\left\{r \eta|0\rangle_{S}\langle 1|+\right.$ H.c. $\}$ is the general form of an ADC ( $p$ and $r$ are the atom's initial populations and coherences). Specifically [1], $G(t)=$ $e^{-\lambda / 2 t}[\cosh (d t / 2)+\lambda / d \sinh (d t / 2)]$ with $d=\sqrt{\lambda^{2}-2 \gamma_{0} \lambda}$. Here, $\lambda$ is the width of $J(\omega)$, while $\gamma_{0}$ is related to the strength of the coupling [1]. The ratio $\gamma_{0} / \lambda$ in fact rules the occurrence of NM effects [32]. For $\lambda \gg \gamma_{0}, J(\omega)$ becomes about flat and $G(t) \rightarrow e^{-\gamma_{0} / 2 t}$ : The atom undergoes standard spontaneous emission at a rate $\gamma_{0}$ and $\dot{\rho} \rightarrow \mathcal{L}[\rho]$, namely, the Markovian regime occurs $(\mathcal{L}$ is the usual zero-temperature atomic Lindbladian [1] with associated rate $\gamma_{0}$ ). For $\lambda<$ $\gamma_{0} / 2$, instead, damped oscillations take place as a signature of non-Markovianity. In particular, in the regime $\lambda \ll \gamma_{0}$, $G(t) \simeq e^{-\lambda t} \cos (\Omega t)$ with $\Omega=\sqrt{\gamma_{0} \lambda / 2}$ showing that the atom undergoes damped Rabi oscillations at a rate $\Omega$ due to its coupling to the cavity protected mode. For $\lambda \simeq 0$ (ideal cavity) we would thus obtain $\mathcal{A}_{G(t)}\left[\rho_{0}\right] \simeq \mathcal{A}_{\cos (\Omega t)}\left[\rho_{0}\right]$. This strongly suggests to regard the cavity protected mode as a generic ancilla in our CM framework and thus set $\mathcal{E}(t) \equiv \mathcal{A}_{\cos (\Omega t)}$ and, additionally, $\Gamma \equiv \lambda$. Indeed, we have shown that if $\Gamma=0$ (namely, $\lambda=0$ ), $S$ behaves as if interacting all the time with one ancilla, namely, the protected mode. On the other hand, we have seen that when $\Gamma$ is very large (Markovian limit) at each collision the system interacts with a fresh ancilla still in the initial state. Note that even this case can be viewed as an effective single-ancilla process if one supposes such ancilla to be reset to its initial state between two next collisions with $S$. Correspondingly, in the atom-field model, for very large $\lambda$ the cavity is bad: The protected-mode leakage is so effective that the atom in fact keeps "seeing" such a mode in its vacuum state at any time. With the above settings $\left[\mathcal{E}(t) \equiv \mathcal{A}_{\cos (\Omega t)}\right.$ and $\Gamma \equiv$ $\lambda]$ the dynamical map $\Lambda(t)$ can be calculated exactly through Eqs. (6)-(8) [33]. Figure 2 shows the atomic excitation, i.e., the excited-state population, and coherences (normalized to the respective initial values) versus time as given by the exact solution (ES) and our CM. For comparison, we also report the corresponding functions predicted by the phenomenological ME (PME) [7] and the SL ME [8] for the MKF $k\left(t^{\prime}\right)=\lambda e^{-\lambda t^{\prime}}$ (a similar study appeared in Ref. [11]). For large $\lambda / \gamma_{0}$ the Markovian regime occurs: All the models basically yield the same purely exponential behavior [see Figs. 2(a) and 2(d)]. As $\lambda$ becomes low, significant deviations arise. The SL model keeps predicting exponential decays [cf. Figs. 2(b), 2(c), 2(e), and 2(f)] in contrast to the damped oscillations predicted by the ES. The PME predicts coherences matching the ES [cf. Figs. 2(e) and 2(f)], yet positivity is drastically violated $[7,11]$ 

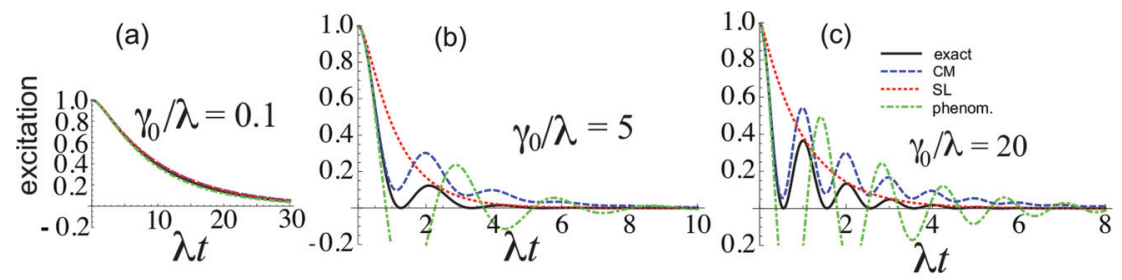

(d)

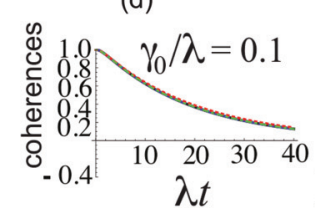

(e)

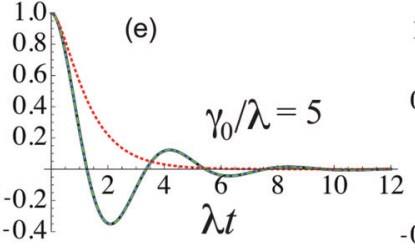

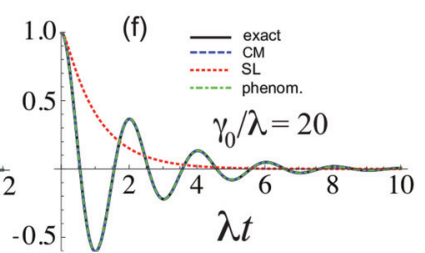

FIG. 2. (Color online) Dynamics of an atom in contact with a bath of Lorentzian spectral density. (a)-(c) [(d)-(f)] show the excitedstate population (coherences) vs the rescaled time $\lambda t$ predicted by the ES (black solid line), our CM (blue dashed), the SL ME (red dotted), and the PME (green dotted-dashed) for different values of $\gamma_{0} / \lambda$. All the plotted quantities are normalized to the respective initial values. [see Figs. 2(b) and 2(c)]. Our ME Eq. (5) yields a substantial improvement on both the above models. As the PME, it accurately reproduces the exact coherences [see Figs. 2(e) and 2(f)]. Quite differently, though, in line with our general proof it is positive [see Figs. 2(b) and 2(c)], a feature shared with the SL ME. Yet, unlike this, the CM captures the physics of the process far better: Damped oscillations for populations rather close to the exact ones are predicted (the discrepancy decreases as $\lambda / \gamma_{0} \rightarrow 0$ ). In Figs. 2(b) and 2(c), while the ES minima are zero, the corresponding CM minima are small but strictly positive. This is likely to stem from the incoherent mixture of identity and swap entering Eq. (1). Using a unitary partial swap, zero minima can indeed occur [27].

Conclusions. We introduced a NM microscopic CM, where the bath memory is added dynamically through simple interancillary collisions each modeled as a swapping operation.
The model interpolates between two extreme situations: a fully Markovian regime and a strongly NM one (where a continuous interaction with a single ancilla occurs). The continuous limit yields an unconditionally CPT ME. To test our approach, we applied it to an atom coupled to a bath of modes featuring a Lorentzian spectral density and compared the outcomes with the analytical solution and two known memory-kernel MEs. While all the advantageous features of such MEs simultaneously occur in ours, this in addition succeeds to capture distinctive traits of the NM dynamics.

Acknowledgments. We thank R. Fazio for discussions and acknowledge support from MIUR through the FIRB-IDEAS project RBID08B3FM and PRIN Project 'Collective quantum phenomena: From strongly correlated systems to quantum simulators'.
[1] H. P. Breuer and F. Petruccione, The Theory of Open Quantum Systems (Oxford University Press, Oxford, UK, 2002).

[2] U. Weiss, Quantum Dissipative Systems, 3rd ed. (World Scientific, Singapore, 2008).

[3] A. Rivas and S. F. Huelga, Open Quantum Systems. An Introduction (Springer, Heidelberg, 2011).

[4] H.-P. Breuer, J. Phys. B 45, 154001 (2012).

[5] See, e.g., J. Schliemann, A. Khaetskii, and D. Loss, J. Phys.: Condens. Matter 15, R1809 (2003); M. Michel, G. Mahler, and J. Gemmer, Phys. Rev. Lett. 95, 180602 (2005).

[6] B. Vacchini and H. P. Breuer, Phys. Rev. A 81, 042103 (2010).

[7] S. M. Barnett and S. Stenholm, Phys. Rev. A 64, 033808 (2001).

[8] A. Shabani and D. A. Lidar, Phys. Rev. A 71, 020101 (2005).

[9] A. A. Budini, Phys. Rev. A 69, 042107 (2004).

[10] S. Daffer, K. Wódkiewicz, J. D. Cresser, and J. K. McIver, Phys. Rev. A 70, 010304(R) (2004).

[11] S. Maniscalco and F. Petruccione, Phys. Rev. A 73, 012111 (2006); S. Maniscalco, ibid. 75, 062103 (2007).

[12] H. P. Breuer and B. Vacchini, Phys. Rev. E 79, 041147 (2009).

[13] J. Wilkie and Y. M. Wong, J. Phys. A 42, 015006 (2009).

[14] S. Campbell et al., Phys. Rev. A 85, 032120 (2012).

[15] D. Chruściński and A. Kossakowski, Phys. Rev. Lett. 104, 070406 (2010); Europhys. Lett. 97, 20005 (2012).
[16] L. Mazzola, E.-M. Laine, H.-P. Breuer, S. Maniscalco, and J. Piilo, Phys. Rev. A 81, 062120 (2010).

[17] H.-P. Breuer, E.-M. Laine, and J. Piilo, Phys. Rev. Lett. 103, 210401 (2009).

[18] J. Rau, Phys. Rev. 129, 1880 (1963).

[19] R. Alicki and K. Lendi, Quantum Dynamical Semigroups and Applications, Lecture Notes in Physics Vol. 717 (Springer, Berlin, 1987).

[20] M. Ziman et al., Phys. Rev. A 65, 042105 (2002); V. Scarani, M. Ziman, P. Štelmachovič, N. Gisin, and V. Bužek, Phys. Rev. Lett. 88, 097905 (2002).

[21] M. Ziman and V. Buzek, Phys. Rev. A 72, 022110 (2005); M. Ziman, P. Štelmachovič, and V. Bužek, Open Syst. Inf. Dyn. 12, 81 (2005).

[22] S. Attal and Y. Pautrat, Ann. Inst. Henri Poincaré 7, 59 (2006).

[23] C. Pellegrini and F. Petruccione, J. Phys. A: Math. Theor. 42, 425304 (2009).

[24] V. Giovannetti and G. M. Palma, Phys. Rev. Lett. 108, 040401 (2012); J. Phys. B 45, 154003 (2012).

[25] T. Rybar, S. N. Filippov, M. Ziman, and V. Bužek, J. Phys. B 45, 154006 (2012).

[26] M. A. Nielsen and I. L. Chuang, Quantum Computation and Quantum Information (Cambridge University Press, Cambridge, UK, 2000). 
[27] F. Ciccarello, G. M. Palma, and V. Giovannetti (unpublished).

[28] We use $\rho$ to denote states of $S$ and $\sigma$ for overall states ( $S$ and the bath).

[29] We are thus assuming that each ancilla is initially in the pure state $|0\rangle$. This is not restrictive: A mixed state can be account for through the known purification picture [26].
[30] See Supplemental Material at http://link.aps.org/supplemental/ 10.1103/PhysRevA.87.040103 for technical details.

[31] B. M. Garraway, Phys. Rev. A 55, 2290 (1997).

[32] E.-M. Laine, J. Piilo, and H.-P. Breuer, Phys. Rev. A 81, 062115 (2010); J.-G. Li, J. Zou, and B. Shao, ibid. 81, 062124 (2010).

[33] F. Ciccarello and V. Giovannetti, Phys. Scr. T 153, 014010 (2013). 